\title{
On the Eigenvectors of a Finite-Difference Approximation to the Sturm-Liouville Eigenvalue Problem
}

\author{
By Eckart Gekeler
}

Abstract. This paper is concerned with a centered finite-difference approximation to to the nonselfadjoint Sturm-Liouville eigenvalue problem

$$
\begin{aligned}
L[u] & =-\left[a(x) u_{x}\right]_{x}-b(x) u_{x}+c(x) u=\lambda u, \quad 0<x<1, \\
u(0) & =u(1)=0 .
\end{aligned}
$$

It is shown that the eigenvectors $w_{p}$ of the $M \times M$-matrix $(\Delta x=1 /(M+1)$ mesh size), which approximates $L$, are bounded in the maximum norm independent of $M$ if they are normalized so that $\left|W_{p}\right|_{2}=1$.

1. Introduction. The present paper is concerned with the nonselfadjoint problem

$$
\begin{aligned}
-\left[a(x) u_{x}\right]_{x}-b(x) u_{x}+c(x) u & =\lambda u, \quad 0<x<1, \\
u(0) & =u(1)=0,
\end{aligned}
$$

where $a(x) \geqslant \alpha>0, c(x) \geqslant 0$, and $a, b, c$ are all bounded and smooth functions. This problem has an infinite sequence of positive and distinct eigenvalues $0<\lambda_{1}<$ $\lambda_{2}<\lambda_{3}<\cdots$ and a corresponding sequence of smooth eigenfunctions $u^{1}, u^{2}, u^{3}, \cdots$ (see, for instance Protter-Weinberger [10, p. 37] and Coddington-Levinson [4, p. 212]). Following Courant-Hilbert $\left[5\right.$, p. 334], the eigenfunctions $u^{p}$ are uniformly bounded in the supremum norm if they are normalized so that

$$
\int_{0}^{1}\left|u^{p}(x)\right|^{2} d x=1, \quad p=1,2,3, \cdots
$$

Of course, by the well-known transformation

$$
u(x)=\exp \left(-1 / 2 \int_{0}^{x} \frac{b(t)}{a(t)} d t\right) w(x)
$$

(1) may be put in the selfadjoint form

Received October 4, 1973.

AMS (MOS) subject classifications (1970). Primary $65 \mathrm{~L} 15$.

Key words and phrases. Sturm-Liouville eigenvalue problem, finite-difference approximation. 


$$
\begin{aligned}
-\left[a(x) w_{x}\right]_{x}+\widetilde{c}(x) w & =\lambda w, \quad 0<x<1, \\
w(0) & =w(1)=0
\end{aligned}
$$

where

$$
\widetilde{c}(x)=c(x)+1 / 2 b_{x}(x)+1 / 4 b^{2}(x) / a(x) .
$$

Here, in order to obtain $\widetilde{c}(x) \geqslant 0$, we have to make a restricting assumption on $b_{x}$. Therefore, we choose the direct approximation of (1) by means of the finite-difference equations

$$
\begin{gathered}
-\frac{a_{k+1 / 2}\left(v_{k+1}-v_{k}\right)-a_{k-1 / 2}\left(v_{k}-v_{k-1}\right)}{\Delta x^{2}}-b_{k} \frac{v_{k+1}-v_{k-1}}{2 \Delta x}+c_{k} v_{k}=\Lambda v_{k}, \\
\quad k=1, \cdots, M, \\
v_{0}=v_{M+1}=0,
\end{gathered}
$$

where $M \in \mathbf{N}, \Delta x=1 /(M+1)$, and $v_{k}=v(k \Delta x)$. Equivalently, we may write (3) in matrix-vector notation

$$
L V=\Lambda V,
$$

where $V=\left(v_{1}, \cdots, v_{M}\right)^{T}$ and the matrix $L$ may be easily derived from (3).

Let $|b(x)| \leqslant \beta$ and $0<\Delta x<2 \alpha / \beta$. Then the matrix $L$ is equivalent to a real symmetric matrix (see Carasso [2]). Using this fact and Theorem 1.8 of Varga [11], it can be shown that all eigenvalues $\Lambda_{p}$ of (3) are real and positive, $0<\Lambda_{1} \leqslant \Lambda_{2} \leqslant$ $\Lambda_{3} \leqslant \cdots \leqslant \Lambda_{M}$, and there exists a complete sequence of corresponding eigenvectors $V^{p}$. A result of Carasso [2, Corollary 1] says that there exist a constant $K$ and an integer $p_{0}$, both independent of $M$, such that

$$
\left|V^{p}\right|_{\infty}=\max _{1 \leqslant k \leqslant M}\left|v_{k}^{p}\right| \leqslant K p^{1 / 2}, \quad p_{0} \leqslant p \leqslant M
$$

if

$$
\left|V^{p}\right|_{2}^{2}=\Delta x \sum_{k=1}^{M}\left|v_{k}^{p}\right|^{2}=1 .
$$

In the selfadjoint case, this result goes back to Bückner [1]. In this paper, we prove the following theorem:

TheOREM. Let $a(x) \geqslant \alpha>0$ and $c(x) \geqslant 0,0<x<1$. Assume that $a, b$, and $c$ are differentiable bounded functions with bounded derivatives. Let $|b(x)| \leqslant \beta$. Let $0<\Delta x \leqslant \alpha / \beta$ and let $\left\{V^{p}\right\}_{p=1}^{M}$ be the eigenvectors of (3), normalized so that $\left|V^{p}\right|_{2}=1$. Then

$$
\left|V^{p}\right|_{\infty} \leqslant \kappa, \quad p=1, \cdots, M,
$$

for some constant $\kappa$ independent of $M$. 
Remark 1. In the case of the equation $u_{x x}=\lambda u$, this result may be proved by explicit computation of the eigenvectors $V^{p}$ (see Isaacson-Keller [9, 9.1.1]).

Applications of the Theorem to the theory of finite-difference approximations to parabolic and hyperbolic partial differential equations are given in [3], [7], [8] .

2. Proof of the Theorem. Instead of $L$, we consider, as in [2], the eigenvectors $D^{-1} V^{p}$ of the similar matrix $D^{-1} L D$ defined below. But, in contrast to Carasso [2] , who uses a discrete maximum principle for his estimation, we then transpose the proof of Courant-Hilbert [5, p. 334] to the resulting discrete problem.

The following basic results are needed.

Lemma 1 (Carasso[2, Lemma 1], [3, Lemma 3.1]). Let $D=\left(d_{1}, \cdots, d_{M}\right)$ be the diagonal matrix with

$$
d_{1}=1, \quad d_{i}=+\left[\prod_{k=1}^{i-1} \frac{a_{k+1 / 2}-b_{k+1} \Delta x / 2}{a_{k+1 / 2}+b_{k} \Delta x / 2}\right]^{1 / 2}, \quad i=2, \cdots, M
$$

For $0<\Delta x<2 \alpha / \beta$, we have $d_{i}>0$ and

$$
|D|_{\infty} \leqslant \kappa_{1}, \quad\left|D^{-1}\right|_{\infty} \leqslant \kappa_{2}
$$

for constants $\kappa_{1}, \kappa_{2}$ independent of $M$. Furthermore, $D^{-1} L D=(P+Q) / \Delta x^{2}$ where $P=\left(p_{i k}\right)_{i, k=1, \cdots, M}$,

$$
\begin{array}{rlrl}
p_{i k} & =\left(p_{k+1 / 2}+p_{k-1 / 2}\right), & & i=k, \\
& =-p_{k+1 / 2}, & & i=k+1, \\
& =-p_{i+1 / 2}, & & k=i+1, \\
& =0, & & \text { otherwise, } \\
p_{k+1 / 2} & =\left(a_{k+1 / 2}-b_{k+1} \Delta x / 2\right)^{1 / 2}\left(a_{k+1 / 2}+b_{k} \Delta x / 2\right)^{1 / 2},
\end{array}
$$

and $Q=\left(q_{1}, \cdots, q_{M}\right)$ is the diagonal matrix with

$$
q_{k}=\left(a_{k+1 / 2}+a_{k-1 / 2}\right)-\left(p_{k+1 / 2}+p_{k-1 / 2}\right)+\Delta x^{2} c_{k}
$$

Remark 2. The change of variables $V=D W$ is a discrete analog to (2) [2]. Lemma 2 (CARAsso [2, Theorem 1]). Let $\Lambda_{p}, V^{p}$ be the characteristic pairs of the matrix $L$ with $\left|V^{p}\right|_{2}=1$. Let $u^{p}$ be an eigenfunction of (1) corresponding to $\lambda_{p}$ and let $U^{p}$ be the vector of dimension $M$ obtained from $u^{p}$ by mesh-point evaluation. Assume $u^{p}$ normalized so that $\left|D^{-1} U^{p}\right|_{2}=\left|D^{-1} V^{p}\right|_{2}$, then, as $\Delta x \rightarrow 0$, we have

$$
\left|\lambda_{p}-\Lambda_{p}\right| \leqslant \kappa_{3}(p) \Delta x^{2}, \quad\left|U^{p}-V^{p}\right|_{2} \leqslant \kappa_{4}(p) \Delta x^{2}
$$


where $\kappa_{3}, \kappa_{4}$ are positive constants depending only on $p$.

In the selfadjoint case, Lemma 2 was proved by Gary [6] .

Remark 3. The estimation (4) implies $\left|U^{p}-V^{p}\right|_{\infty} \leqslant \kappa_{4}(p) \Delta x^{3 / 2}$.

LEMMA 3. Let

$$
C_{l}(W)=\sum_{k=1}^{l}\left[p_{k+1 / 2}\left(w_{k}-w_{k+1}\right)-p_{k-1 / 2}\left(w_{k}-w_{k-1}\right)\right] q_{k} w_{k} / \Delta x^{2}
$$

Then, under the assumptions of the Theorem,

$$
C_{l}\left(W^{p}\right)=-q_{l} p_{l+1 / 2} w_{l+1}^{p} w_{l}^{p} / \Delta x^{2}+O(1), \quad l=1, \cdots, M,
$$

where $W^{p}=D^{-1} V^{p}$ and $O(1)$ denotes a function which has a bound independent of $M$.

Proof. We show at first that $\left|q_{k} / \Delta x^{2}\right| \leqslant \kappa_{5}$ independently of $M$. To this end, it suffices to consider $a_{k+1 / 2}-p_{k+1 / 2}$. By means of the binomial theorem, we obtain

$$
\begin{aligned}
a_{k+1 / 2} & -p_{k+1 / 2} \\
= & a_{k+1 / 2}-a_{k+1 / 2}\left(1-\frac{b_{k+1} \Delta x}{4 a_{k+1 / 2}}+O\left(\Delta x^{2}\right)\right)\left(1+\frac{b_{k} \Delta x}{4 a_{k+1 / 2}}+O\left(\Delta x^{2}\right)\right)
\end{aligned}
$$

Inserting $b_{k+1}=b_{k}+O(\Delta x)$, we find that

$$
a_{k+1 / 2}-p_{k+1 / 2}=O\left(\Delta x^{2}\right) .
$$

Now, since $w_{0}^{p}=0$,

$$
\begin{aligned}
C_{l}\left(W^{p}\right)= & -q_{l} p_{l+1 / 2} w_{l+1}^{p} w_{l}^{p} / \Delta x^{2} \\
& +\sum_{k=1}^{l} \frac{q_{k}}{\Delta x^{2}}\left(p_{k+1 / 2}-p_{k-1 / 2}\right) w_{k}^{p} w_{k}^{p}+\sum_{k=1}^{l-1} \frac{q_{k+1}-q_{k}}{\Delta x^{2}} p_{k+1 / 2} w_{k+1}^{p} w_{k}^{p} .
\end{aligned}
$$

But, by the mean value theorem, we have $p_{k+1}-p_{k}=O(\Delta x)$ and $\left(q_{k+1}-q_{k}\right) / \Delta x^{2}=O(\Delta x)$. Hence, using Schwarz's inequality and $\left|W^{p}\right|_{2} \leqslant \kappa_{6}$, we obtain the desired result.

Now, according to Lemma 1 , it suffices to prove the Theorem for the eigenvectors $W^{p}=D^{-1} V^{p}$ of the matrix $(P+Q) / \Delta x^{2}$ which also has the eigenvalues $\Lambda_{p}$. We multiply the $k$ th row of

$$
\left(1 / \Delta x^{2}\right)(P+Q) W^{p}=\Lambda_{p} W^{p}
$$

by 


$$
p_{k+1 / 2}\left(w_{k}^{p}-w_{k+1}^{p}\right)-p_{k-1 / 2}\left(w_{k}^{p}-w_{k-1}^{p}\right)
$$

and obtain, by adding all rows from $k=1$ to $k=l$,

$$
\left[\frac{p_{l+1 / 2}\left(w_{l}^{p}-w_{l+1}^{p}\right)}{\Delta x}\right]^{2}+C_{l}\left(W^{p}\right)
$$

$$
+\Lambda_{p} p_{l+1 / 2} w_{l+1}^{p} w_{l}^{p}-\Lambda_{p} \Delta x \sum_{k=1}^{l} p_{x}\left(\xi_{k}\right) w_{k}^{p} w_{k}^{p}=\left[\frac{p_{1 / 2}\left(w_{1}^{p}-w_{0}^{p}\right)}{\Delta x}\right]^{2}
$$

where $(k-1 / 2) \Delta x<\xi_{k}<(k+1 / 2) \Delta x$. In order to eliminate the term on the right side of (6), we sum up Eqs. (6) for $l=1$ to $l=M$, add $\left(p_{1 / 2}\left(w_{1}^{p}-w_{0}^{p}\right) / \Delta x\right)^{2}$ to both sides, and divide by $M+1=1 / \Delta x$. Then

$$
\left[\frac{p_{1 / 2}\left(w_{1}^{p}-w_{0}^{p}\right)}{\Delta x}\right]^{2}=\Delta x \sum_{l=0}^{M}\left[\frac{p_{l+1 / 2}\left(w_{l+1}^{p}-w_{l}^{p}\right)}{\Delta x}\right]^{2}+\Delta x \sum_{l=1}^{M} C_{l}\left(W^{p}\right)
$$

$$
+\Lambda_{p} \Delta_{x} \sum_{l=1}^{M} p_{l+1 / 2} w_{l+1}^{p} w_{l}^{p}-\Lambda_{p} \Delta x^{2} \sum_{l=1}^{M} \sum_{k=1}^{l} p_{x}\left(\xi_{k}\right) w_{k}^{p} w_{k}^{p}
$$

But

$$
0<\alpha / 2<p_{l+1 / 2}<\kappa_{7} \quad\left(\kappa_{7}>1\right)
$$

if $0<\Delta x \leqslant \alpha / \beta$. Thus, using the fundamental relation

$$
\sum_{l=0}^{M} p_{l+1 / 2}\left(w_{l+1}-w_{l}\right)^{2}=W^{T} P W
$$

$\left(w_{0}=w_{M+1}=0\right)$, we derive

$$
\begin{gathered}
\Delta x \sum_{l=0}^{M}\left[\frac{p_{l+1 / 2}\left(w_{l+1}^{p}-w_{l}^{p}\right)}{\Delta x}\right]^{2} \leqslant \Delta x \kappa_{7}\left(W^{p}\right)^{T} P W^{p} / \Delta x^{2} \\
\quad=\Delta x \kappa_{7}\left(W^{p}\right)^{T}(P+Q) W^{p} / \Delta x^{2}-\Delta x \kappa_{7} \sum_{l=1}^{M} q_{l} w_{l}^{p} w_{l}^{p} / \Delta x^{2} \\
\leqslant \Delta x \kappa_{7} \Lambda_{p}\left(W^{p}\right)^{T} W^{p}+\kappa_{5} \kappa_{7} \Delta x\left(W^{p}\right)^{T} W^{p}=\kappa_{7} \Lambda_{p}+\kappa_{5} \kappa_{7},
\end{gathered}
$$

since $\left|q_{l} / \Delta x^{2}\right| \leqslant \kappa_{5}$ independently of $M$ and $\left|W^{p}\right|_{2}^{2}=1$. Hence, applying Schwarz's inequality, we find from (7) by means of the assumption and Lemma 3 that

$$
\left[p_{1 / 2}\left(w_{1}^{p}-w_{0}^{p}\right) / \Delta x\right]^{2} \leqslant \kappa_{8} \Lambda_{p}+\kappa_{9}
$$


From this estimation, Eq. (6), and Lemma 3, we deduce that

$$
\left(\Lambda_{p}-q_{l} / \Delta x^{2}\right) p_{l+1 / 2} w_{l+1}^{p} w_{l}^{p} \leqslant \kappa_{10} \Lambda_{p}+\kappa_{11} .
$$

Consequently, observing (8), we obtain, in case $\Lambda_{p}>\kappa_{5}$, that

$$
w_{l+1}^{p} w_{l}^{p} \leqslant \kappa_{12}, \quad l=1, \cdots, M,
$$

for some constant $\kappa_{12}$ independent of $M$. For $\Lambda_{p} \leqslant \kappa_{5}$, the assertion of the Theorem follows by Lemma 2, Remark 3.

Finally, we return once more to Eq. (6). The above estimation yields

$$
\left[\frac{p_{l+1 / 2}\left(w_{l+1}^{p}-w_{l}^{p}\right)}{\Delta x}\right]^{2} \leqslant \kappa_{13} \Lambda_{p}+\kappa_{14}, \quad l=1, \cdots, M,
$$

or, using (9),

$$
\begin{aligned}
\left|w_{l}^{p}\right|^{2} & \leqslant\left|w_{l}^{p}\right|^{2}+\left|w_{l+1}^{p}\right|^{2}=\left(w_{l+1}^{p}-w_{l}^{p}\right)^{2}+2 w_{l}^{p} w_{l+1}^{p} \\
& =\frac{\Delta x^{2}}{p_{l+1 / 2}^{2}}\left[\frac{p_{l+1 / 2}\left(w_{l+1}^{p}-w_{l}^{p}\right)}{\Delta x}\right]^{2}+2 w_{l+1}^{p} w_{l}^{p} \\
& \leqslant \Delta x^{2}\left(\kappa_{15} \Lambda_{p}+\kappa_{16}\right)+\kappa_{17}=\kappa, \quad l=1, \cdots, M .
\end{aligned}
$$

Hence, $\max _{1 \leqslant p \leqslant M}\left\{\left|W^{p}\right|_{\infty}\right\}$ is bounded independently of $M$.

Mathematics Department

Universität Stuttgart

7000 Stuttgart, Germany

1. H. BÜCKNER, "Über Konvergenzsätze, die sich bei der Anwendung eines Differenzenverfahrens auf ein Sturm-Liouvillesches Eigenwertproblem ergeben," Math. Z., v. 51, 1944, pp. 423465. MR 11, 58.

2. A. CARASSO, "Finite-difference methods and the eigenvalue problem for nonselfadjoint Sturm-Liouville operators," Math. Comp., v. 23, 1969, pp. 717-729. MR 41 \#2938.

3. A. CARASSO \& S. V. PARTER, An analysis of 'boundary-value techniques' for parabolic problems," Math. Comp., v. 24, 1970, pp. 315-340. MR 44 \#1249.

4. E. A. CODDINGTON \& N. LEVINSON, Theory of Ordinary Differential Equations, McGraw-Hill, New York, 1955. MR 16, 1022.

5. R. COURANT \& D. HILBERT, Methoden der Mathematischen Physik. Vol. 1, Interscience, New York, 1953. MR 16, 426.

6. J. GARY, "Computing eigenvalues of ordinary differential equations by finite differences," Math. Comp., v. 19, 1965, pp. 365-379. MR 31 \#163.

7. E. GEKELER, "Long-range and periodic solutions of parabolic problems," Math. Z., v. 134,1973 , pp. 53-66.

8. E. GEKELER, “ $A$-convergence of finite-difference approximations of parabolic initial boundary value problems," SIAM J. Numer. Anal. (To appear.) 
9. E. ISAACSON \& H. B. KELLER, Analysis of Numerical Methods, Wiley, New York, 1966. MR 34 \#924.

10. M. H. PROTTER \& H. F. WEINBERGER, Maximum Principles in Differential Equations, Prentice-Hall, Englewood Cliffs, N. J., 1967. MR 36 \#2935.

11. R. S. VARGA, Matrix Iterative Analysis, Prentice-Hall, Englewood Cliffs, N. J., 1962. MR 28 \#1 725. 